\title{
Peripheral aberrations in the human eye for different wavelengths: off-axis chromatic aberration
}

\author{
Bart Jaeken, ${ }^{1, *}$ Linda Lundström, ${ }^{2}$ and Pablo Artal ${ }^{1}$ \\ ${ }^{1}$ Laboratorio de Optica, Instituto Universitario de Investigación en Óptica y Nanofísica (IUiOyN), Universidad de Murcia, \\ Campus de Espinardo (Ed. 34), 30100 Murcia, Spain \\ ${ }^{2}$ Biomedical and X-Ray Physics, Royal Institute of Technology, 10044 Stockholm, Sweden \\ *Corresponding author: bart.jaeken@um.es
}

Received June 17, 2011; revised July 25, 2011; accepted July 25, 2011;

posted July 25, 2011 (Doc. ID 149483); published August 25, 2011

\begin{abstract}
The interest in the eye's off-axis aberrations has increased strongly. On-axis the conversion of the aberration magnitude between different wavelengths is well known. We verified if this compensation is correct also for off-axis measurements by building a wavelength tunable peripheral Hartmann-Shack sensor and measuring 11 subjects out to $\pm 30^{\circ}$ in the horizontal visual field. At the fovea, an average longitudinal chromatic aberration of $1 \mathrm{D}$ between red $(671 \mathrm{~nm})$ and blue $(473 \mathrm{~nm})$ light was found, and it increased slightly with eccentricity (up to $1.2 \mathrm{D})$. A similar trend was measured for astigmatism as a function of wavelength (increase $\sim 0.15 \mathrm{D}$ ). Computational ray tracing in model eyes showed that the origin of the small increase of chromatic aberrations with eccentricity is the change of the oblique power of the refractive surfaces in the eye. Factors related to increase of axial length and refractive index of the eye were found to have a very small influence. (c) 2011 Optical Society of America
\end{abstract} OCIS codes: $\quad 330.0330,120.4570$.

\section{INTRODUCTION}

There is a growing interest in the off-axis image quality of the human eye, mainly due to the suggestion that refractive errors in the peripheral field might be a cue for emmetropization. Some earlier studies on animals have shown that the growth of the eye can be experimentally manipulated by inducing defocus in eyes of animal models [1] and that peripheral defocus might play an important role in the emmetropization process [2-5]. Some evidence has accumulated that similar mechanisms operate also in human eyes [6]. Various studies have shown that myopes tend to have their peripheral focal plane more behind the retina, relative to the fovea, while emmetropes have it more in front of the retina [7-13]. The relationship between optical features of the eye and visual function in the periphery has also been investigated $[14,15]$.

The optics in the periphery of the human eye have been measured objectively using techniques that analyze the reflection from the retina, including the double-pass technique $[\underline{13}, 16,17]$ or the Hartmann-Shack wavefront sensor $[\underline{9}, \underline{18}, \underline{19}]$. These measurements are most often performed in quasimonochromatic light in the near-infrared since light of these wavelengths does not stimulate pupil constriction and is more comfortable for the subject. Furthermore, fundus reflectance is higher in infrared than in visible light. Recently, it was shown that even longer wavelengths (up to $1070 \mathrm{~nm}$ ), totally invisible, can be used for optical measurements in the human eye [20].

An important question when measuring with light of different wavelengths is how ocular aberrations change. For the fovea, this has been extensively studied and published. The main changes occur for defocus and the dioptric difference for light from the two ends of the visible spectrum represents the longitudinal chromatic aberration (LCA). LCA is quite similar among subjects. This has been shown with both objective [21] and subjective methods [22]. Thibos et al. [23] suggested that the eye's LCA could be modeled with an equation based on Cornu's hyperbolic formula for the refraction of water but with coefficients adjusted for the human eye. Atchison and Smith [24] proposed a Cauchy dispersion equation, which gives slightly larger chromatic differences in the long wavelength range. Nam et al. [25] recently suggested a method of backward ray tracing in a one-surface model eye to convert between wavelengths. This method not only accounts for defocus but for all aberrations.

Chromatic aberration has not yet been described in detail as a function of eccentricity. One reason for this could be that it is commonly assumed that the LCA does not change with eccentricity since the optical path length of the chief ray is very similar on-axis as off-axis. However, chromatic difference is not only caused by the difference in optical path length but also by the difference in refractive power of the various surfaces [26]. This power is angle-dependent (also referred to as oblique refractive power [27]) and off-axis Seidel aberrations depend on both the refractive index and the angle of eccentricity. To the best of our knowledge, only one previous study explored off-axis LCA [28] by measuring the chromatic difference in focus along the horizontal meridian of the eye up to $40^{\circ}$ off-axis, using a double-pass method with four wavelengths: 458, 501.8, 543.5, and $632.8 \mathrm{~nm}$. Four subjects were measured, resulting in a mean foveal LCA of about $1 \mathrm{D}$ that increased with eccentricity up to $1.5 \mathrm{D}$ at $40^{\circ}$, but the increase was not statistically significant. Further, a simulation in a model eye [29] found good agreement between experimental [28] and simulated data, although the chromatic aberration was different in object and image space.

It is important to know if, and how, chromatic aberration changes with angle of eccentricity in the visual field to compare to different studies. Moreover, such data would permit 
extrapolation to wavelengths outside the measured range. The studies listed above indicate that LCA may vary with eccentricity, but this fact needs further experimental confirmation. In the current study, we addressed how LCA changes with eccentricity and how it affects higher-order aberrations. We therefore built a wavelength tunable peripheral HartmannShack wavefront sensor and measured the chromatic aberrations as a function of eccentricity in 11 subjects. We employed a model eye to simulate our experimental results.

\section{METHODS}

\section{A. Instrument}

We have designed and built a wavelength tunable peripheral Hartmann-Shack (HS) wavefront sensor with an open field of view. A schematic diagram of the system is depicted in Fig. 1. An RGB laser (RGB-671, Changchun New Industries Optoelectronics Technology Co. Ltd, Changchun, China) emitting in three wavelengths: $473 \mathrm{~nm}$ (blue), $532 \mathrm{~nm}$ (green), and $671 \mathrm{~nm}$ (red) was used as a light source. The intensity of the light entering the eye was varied between 0.5 and $1 \mu \mathrm{W} / \mathrm{cm}^{2}$ depending on the wavelength to keep the registered images with a similar signal. The diameter of the beam entering the eye was $1 \mathrm{~mm}$. Achromatic lenses were used to minimize the chromatic aberrations of the system. The combination of dim light in the room, a small entrance beam with a low intensity, and the use of a shutter allowed us to measure the subjects without cycloplegia. The HS spot pattern was imaged onto a highsensitivity electron multiplying CCD (EMCCD) camera (Luca, Andor Technology plc, Belfast, UK) with a magnification of 0.5 .

The fixation target was created by aiming a red laser pointer onto a white piece of plastic that was located for all eccentricities at $1.3 \mathrm{~m}$ from the subject. The target was placed $1^{\circ}$ above the horizontal meridian, which was sufficient to prevent the corneal reflections from entering the measuring arm of the instrument and disturbing the HS measurements. This implies that the results shown here are for the retina $1^{\circ}$ superior to the horizontal meridian. The small mirror in front of the measured eye blocked the vision of the foveal target $\left(0^{\circ}\right)$ but because all measurements were done binocularly, none of the subjects had difficulties fixating at this angle using the other eye.

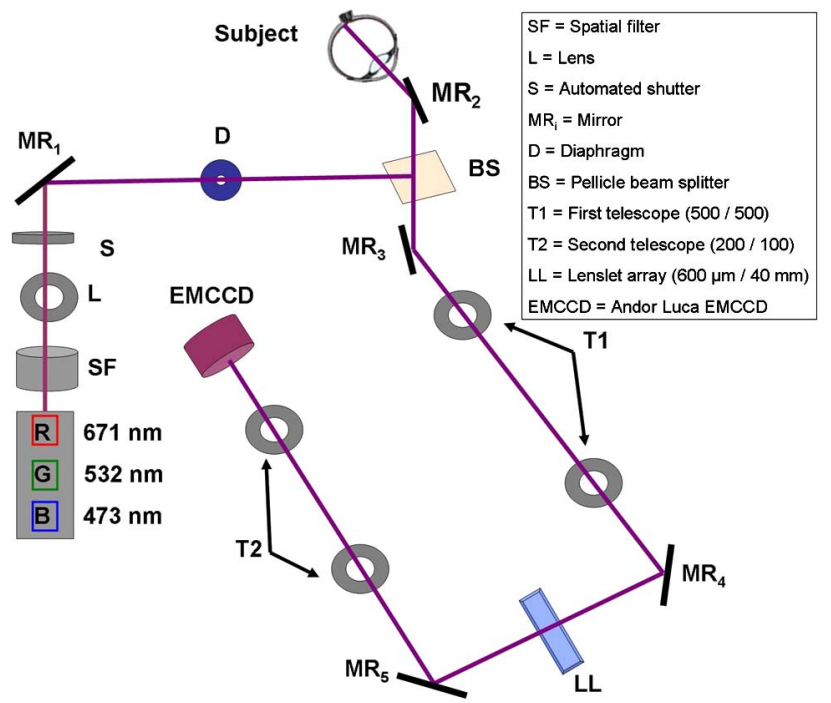

Fig. 1. (Color online) Schematic overview of the instrument setup.
We attempted to take all wavefront measurements in the pupil plane of the subject having the intersection of the visual axis with the pupil plane as center of the wavefront. A pupil camera (not shown in Fig. 1) placed in an angle to the measurement axis of the sensor was used to position the subject in a bite-bar. During calibration the center of the camera was aligned with the cross section of the incoming light and the pupil plane of the system. Once the measuring light entered the subject's eye ( $X-Y$ alignment), the subject was moved in the $Z$ direction to the point where the pupil was centered to the pupil camera. By using this method, the alignment of the subject improved significantly compared to the method where the depth position was determined by the sharpness of the pupil edge. This alignment was only done once for each subject. For off-axis measurements the subject turned the eyes to fixate the target and only small sideway alignments in pupil position were needed between measurements at different eccentricities. An advantage of the system design was that the entrance path of all three different wavelengths was equal. However, one source of error could be the transverse chromatic aberrations (TCA) that cause the entrance beam to be refracted slightly differently for the various wavelengths, creating point sources at a slightly different location. This difference increases with eccentricity but stays very small even at large degrees, i.e., less than 10 arcmin at $30^{\circ}$ off-axis [30]. Because the variation of aberrations with eccentricity in the human eye is gradual, the error caused by measuring at slightly different locations was neglected. To verify this assumption, the impact of the TCA was examined in the simulations (see Results section below). The repeatability error of the sensor was measured in an artificial eye and can be neglected as an error source compared to the dynamics of the living eye. The aberrations present in the system were removed from the measurements by taking a reference image for each color and using it in the elaboration of the data. The residual LCA of the system was smaller than $0.01 \mathrm{D}$.

\section{B. Measuring Procedure and Subjects}

The right eyes of five emmetropes (refractive error (mean \pm std: $-0.38 \pm 0.35 \mathrm{D}$ ), age:30 \pm 2.5 years) and six mild myopes (refractive error: $-2.91 \pm 1.57 \mathrm{D}$, age: $31 \pm 3.0$ years) were measured. Measurements were done with the natural pupil of the subject. No refractive correction was necessary since all subjects had refractive errors within the measurement range of the system. At each of the nine eccentricities $\left(0 \pm 40^{\circ}\right.$ in steps of $\left.10^{\circ}\right)$ three measurements were taken for each of the three wavelengths. The angles used throughout the article represent the visual angle in degrees. Negative angles represent nasal retina and positive angles represent temporal retina. The order of the colors was varied between the subjects as well as the order of eccentricity (measuring from nasal to temporal or from temporal to nasal). All three wavelength cases were measured consecutively in each subject. The total time of the whole procedure was in the order of 20 minutes. Only the short-term variation caused by variation in the tear film, the eye saccades, and small fluctuations in the accommodative state will have impact on the measurements, but this is random also within wavelength and, thus, expected not to bias the data. An estimation of the size of this variation was made by examining the standard deviation of the repeated measurements taken at each investigated combination 
(subject/wavelength/eccentricity). The variation with wavelength and eccentricity were statistically tested using a repeated measures ANOVA test. The spot pattern of one measurement was the average of three frames taken consecutively, each with $200 \mathrm{~ms}$ exposure time. To improve the signalto-noise ratio, a background image was taken before each series of measurements and wavelength. This background image was obtained in the same way as a normal measurement but without the subject in place. It contained the background light of the room and some of the backscattered light from the beam splitter. The use of the wavefront sensor and the experiment followed the tenets of the Declaration of Helsinki. All subjects were fully informed before participation in the study.

\section{Data Analysis}

The spot pattern was processed with an iterative algorithm that uses extrapolated B-spline polynomials to estimate the position of the next neighbor spot (described in detail in [31]). A reference image, obtained by using a larger beam diameter and placing a mirror in the pupil plane of the system, was used to remove aberrations due to the instrument. The derivatives of Zernike polynomials up to the fourth order were fitted to the difference in location between the measured spots and their reference location, using a least-square fitting algorithm. The Zernike coefficients ordering and normalization followed the Optical Society of America Standardization committee recommendations [32]. When measuring off-axis, the shape of the projected pupil becomes elliptical. Initially the polynomials were fitted to a large unit circle enclosing all measured spots. This method uses all measured data points of the natural pupil to fit the wavefront giving a full description of the original wavefront. To facilitate comparison between measurements, the coefficients were then mathematically rescaled to a pupil of $4 \mathrm{~mm}$. A measurement was only used for further analysis if the smallest diameter of the original pupil size was larger than $4 \mathrm{~mm}$. For that reason we had to discard the measurements taken at $40^{\circ}$ off-axis. The variation of chromatic aberrations as function of eccentricity was examined with repeated measures ANOVA tests with within-subject variables "wavelength" and "eccentricity". When appropriate, the sphericity assumption was checked using the estimated epsilon $(\varepsilon)$ value. When the sphericity criterion was not reached, $\varepsilon<0.8$, the degrees of freedom were corrected when calculating the $p$ value. When a correction was used it is indicated in the text along with the $p$ value.

\section{Computer Simulations}

To examine the chromatic aberrations as a function of eccentricity under controlled conditions, computer simulations were conducted using optical analysis and design software (Zemax Development Corporation, Bellevue, Washington, USA). We chose an anatomically correct model eye since the refraction of light of different wavelengths depends on the dimensions, curvatures, and dispersion of the various eye components. The Liou and Brennan model eye [33] was chosen because it contains a gradient index (GRIN) lens. To reproduce the well-known on-axis LCA the dispersion functions suggested by Atchison and Smith [24] were adopted. To validate the model, its off-axis aberrations were compared with the mean off-axis aberrations of the measured emmetropic subjects at green light $(532 \mathrm{~nm})$. Good agreement was found except that our model eye is rotationally symmetric while our measurements showed the known variations between nasal and temporal aberrations, which mainly originate from the difference between the optical and visual axis in the human eye creating an asymmetric variation with eccentricity for astigmatism and coma with larger absolute values for the temporal retina [9,34]. A wavefront sensor assumes that the entrance light creates a point source on the retina and the aberrations are defined as the difference between a plane wavefront and the wavefront coming from that point source after passing the optics of the eye. This was reproduced in the simulations by flipping the model eye, having the retina as object plane. Flipping the eye has an impact on how the GRIN is defined (a detailed overview of the adjusted coefficients is given in Appendix A). The stop was a $7 \mathrm{~mm}$ square whose center was placed in the $X Y$ plane at $Z$ equal to the position of the outer most surface of the eye (the vertex of the cornea). This is important to insure that the wavefront calculations are done equally at each eccentricity. When going off-axis, three parameters were changing: the field angle (FA), which represents the angle of the principal ray inside the eye after refraction, and the decentration, and the tilt of the last two components (stop and image plane). The tilt of the stop represents the off-axis angle, which was set to be similar to those of the experiments (from $0^{\circ}$ to $\pm 30^{\circ}$ in steps of $10^{\circ}$ ). To find the corresponding decentration and FA for a certain angle, the first step was to adjust the FA until the chief ray coincided with the titled optical axis. The decentration of the stop surface was then adjusted until the chief ray passed through the center of the pupil. The settings used in the simulation are for a reference wavelength of $550 \mathrm{~nm}$ and can be found in Appen$\operatorname{dix}$ A. To obtain the wavefront in the same way as in the actual measurements, i.e., vignetted by the iris ( $5 \mathrm{~mm}$ in the model), it is important that the stop is larger than the size of the beam leaving the eye. The calculated wavefront map $(128 \times 128$ pixels) was used as input for calculating the Zernike coefficients. The same protocol as described earlier for the experimental measurements was replicated in the simulations.

\section{RESULTS}

The change with wavelength was evaluated for the secondorder to fourth-order Zernike coefficients as well as the RMS wavefront error. Here we present the mean spherical equivalent (M), horizontal astigmatism (J0) (both in diopters, calculated from $C_{2}^{0}$ and $C_{2}^{2}$ ), horizontal coma $\left[\operatorname{comaH}\left(C_{3}^{1}\right)\right]$, spherical aberration (Sph_Ab $\left(C_{4}^{0}\right)$ ), and the higher-order (third and fourth) RMS value (high_RMS), all given for a $4 \mathrm{~mm}$ circular pupil. The chromatic difference in defocus (M) is referred to as LCA.

The mean and variation of the standard deviations of the three repeated measurements for all eccentricities, wavelengths, and subjects was calculated as a metric of repeatability of the instrument. Over the whole population it was $0.085 \pm 0.058 \mathrm{D}, \quad 0.036 \pm 0.032 \mathrm{D}, \quad 0.011 \pm 0.015 \mu \mathrm{m}, \quad 0.005 \pm$ $0.004 \mu \mathrm{m}$, and $0.010 \pm 0.011 \mu \mathrm{m}$ for, respectively, M, J0, coma_H, Sph_Ab, and high_RMS. Because of the increase of the magnitude of the aberrations with eccentricity, this standard deviation increased slightly, but significantly, as a function of angle for all parameters except for M. The largest values were found at $30^{\circ}$. The average (nasal, temporal) values at $30^{\circ}$ were $0.099 \pm 0.077 \mathrm{D}(\mathrm{M}), 0.050 \pm 0.043 \mathrm{D}(\mathrm{J} 0)$, 
Table 1. Overview of the $p$ Values Comparing the Chromatic Variation Between Blue and Red

\begin{tabular}{lccccc}
\hline & Foveal $^{a}$ & ECC $^{b}$ & $10^{\circ c}$ & $20^{\circ c}$ & $30^{\circ c}$ \\
\hline M $^{d}$ & 0.000 & $0.000^{e}$ & 0.005 (N:0.013/T:0.911) & 0.000 (N:0.018/T:0.000) & 0.003 (N:0.075/T:0.001) \\
J0 & 0.603 & 0.009 & $0.273(\mathrm{~N}: 0.112 / \mathrm{T}: 0.688)$ & 0.037 (N:0.006/T:0.043) & 0.028 (N:0.015/T:0.021) \\
Coma_H & 0.217 & $0.288^{e}$ & $0.144^{e}$ & $0.235^{e}$ & 0.382 \\
Sph_Ab & 0.264 & $0.199^{e}$ & 0.267 & 0.976 & 0.333 \\
high_RMS & 0.001 & $0.448^{e}$ & 0.081 & 0.575 & 0.441 \\
\hline
\end{tabular}

${ }^{a}$ The column "foveal" indicates which on-axis parameters are significantly different with wavelength.

${ }^{b}$ The column "ECC" shows if the chromatic variation is significantly different with eccentricity.

${ }^{c}$ These columns indicate if the mean chromatic difference at the indicated eccentricity is significantly different than the mean foveal chromatic difference.

${ }^{d}$ For $\mathrm{M}$ and $\mathrm{J} 0$, besides the overall $p$ value for the indicated angle, also the specific nasal (N) and temporal (T) $p$ value is given.

${ }^{e}$ Indicates the use of Huynh-Feldt correction when calculating the $p$ value.

$0.015 \pm 0.021 \mu \mathrm{m}$ (coma_H), $0.006 \pm 0.005 \mu \mathrm{m}$ (Sph_Ab), and $0.015 \pm 0.016 \mu \mathrm{m}$ (high_RMS). No variation with wavelength was found. The main part of the variation described by the standard deviation of the three trial measurements finds its origin in the dynamics of the human eye as described above and, therefore, is random between the trials. A repeated measures ANOVA test with the trials as within-subject variable confirmed that they were not significantly different: $p$ values 0.212 (M), 0.768 (J0), 0.602 (coma_H), 0.379 (Sph_Ab), and 0.511 (high_RMS). The mean value of the three measurements is used in the remaining analysis.

The mean and standard deviation of the on-axis (taken at $\left.0^{\circ}\right)$ LCA between red $(671 \mathrm{~nm})$ and blue $(473 \mathrm{~nm})$ was $0.97 \pm$ $0.13 \mathrm{D}$ across all 11 subjects. When estimating the LCA with the dispersion equations [23,24] we found similar values, $\sim 1.06 \mathrm{D}$, within the standard deviation of our measurements. We also examined the wavelength dependence of the on-axis aberrations using a repeated measures ANOVA test with within-subject variable "wavelength" (blue and red). Only M and high_RMS were found significantly different $(p<0.05)$, as can be seen in Table 1 column "foveal". Although high_RMS was found significantly different the difference was very small and, therefore, only chromatic difference in defocus is of practical interest.

\section{A. Peripheral LCA}

Figure 2 shows the mean of defocus (M calculated from $C_{2}^{0}$ for a $4 \mathrm{~mm}$ pupil) for the five emmetropic subjects as a function of eccentricity. The error bars represent the standard deviation between the five subjects. The minus sign in this graph indicates myopia, i.e., the eye is more myopic for blue than for red

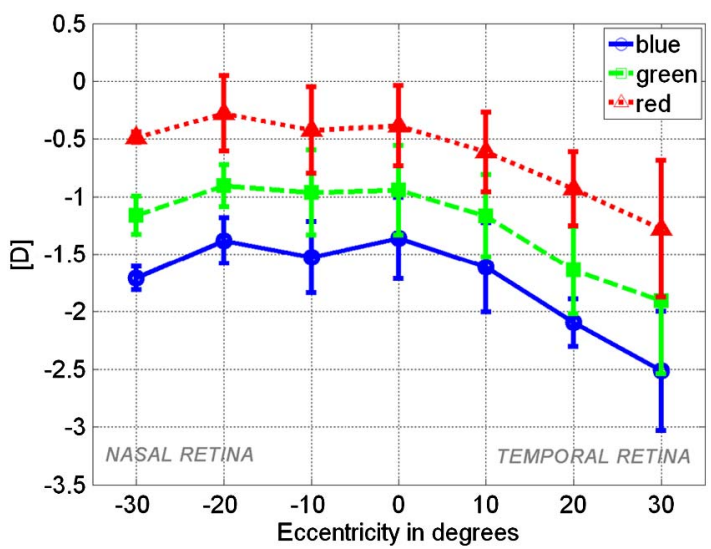

Fig. 2. (Color online) Average values of mean spherical equivalent (M) for the five emmetropes. The error bars represent the standard deviation of $\mathrm{M}$ between the subjects. light. There is an overall trend of relative myopia toward the periphery in agreement with earlier studies on emmetropic subjects [7-9, 12, 13, 34$]$.

The difference in LCA between emmetropes and myopes was examined with a repeated measures ANOVA test with within-subject variable the wavelength (red and blue) and with between-subject variable the subject's refractive group. No significant difference was found ( $p=0.939$ ) between emmetropes and myopes. Figure 3 shows the average LCA for both groups and for the whole population. Here, the blue values at each eccentricity have been subtracted from the red to more clearly show the off-axis LCA.

A small increase of the average LCA with eccentricity (from $0.97 \mathrm{D}$ on-axis to approximately $1.22 \mathrm{D}$ at $30^{\circ}$ ) was observed. A repeated measures ANOVA test was used to test if the off-axis chromatic difference (red-blue) was significantly different compared to the on-axis chromatic difference. Table 1 gives a detailed overview of the $p$ values. The chromatic variation between blue and red was found to be significantly different $(p<0.05)$ with eccentricity. All angles were also examined independently, finding significant difference between the foveal chromatic variation and for the off-axis angles $10^{\circ}$ to $20^{\circ}$ nasally and $20^{\circ}$ to $30^{\circ}$ temporally. The result of the simulated LCA (red-blue) of the model eye is plotted in Fig. 3 and shows good agreement with the measured data (except for the nasal-temporal asymmetry as mentioned earlier) with a similar increase with eccentricity, from $1.04 \mathrm{D}$ on-axis to $1.16 \mathrm{D}$ at $30^{\circ}$.

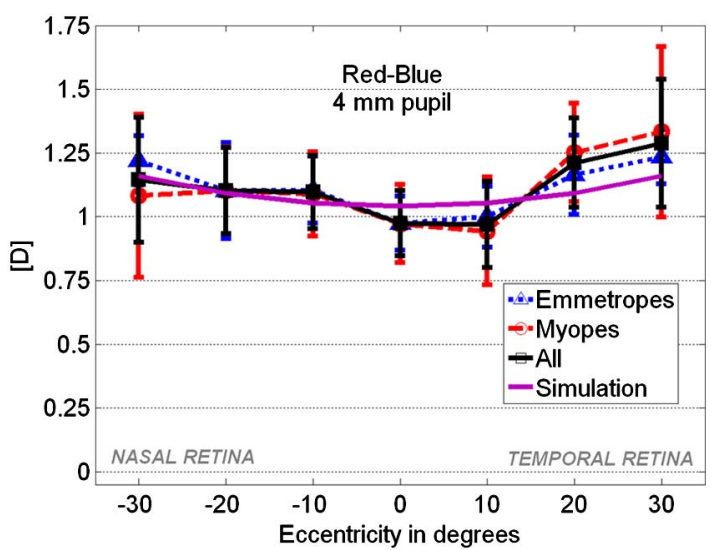

Fig. 3. (Color online) Longitudinal chromatic aberration (MredMblue) as a function of eccentricity for the two refractive groups separately (emmetropes, myopes) and the two combined (all). The error bars give the standard deviation of the calculated LCA between the different subjects. Also, the LCA found in the model eye (simulation) is plotted. 


\section{B. Off-Axis Chromatic Aberrations}

The same analysis as for the off-axis LCA was performed for the other aberrations (J0, coma_H, Sph_ab, and high_RMS). Also here no significant difference was found between the two refractive groups except for high_RMS [ $p$ values: 0.405 (J0), 0.563 (coma_H), 0.349 (Sph_ab), 0.003 (high_RMS)]. The averaged results for all subjects of the chromatic variation of astigmatism and higher-order aberrations as a function of eccentricity are shown in Fig. 4(a) (for clarity, M is not included in the figure because of its large chromatic difference). The standard deviation between the subjects is represented by the error bars. The variation of chromatic difference with eccentricity was found to be significant for J0 $(p=0.009)$ with an average of $0.156 \mathrm{D}\left(0.064 \mu \mathrm{m}\right.$ for $4 \mathrm{~mm}$ pupil) between $0^{\circ}$ and $30^{\circ}$. A detailed analysis (results see Table 1) showed a significant difference of chromatic aberration in the fovea, for both $20^{\circ}$ and $30^{\circ}$, and both in the nasal and temporal retina. The chromatic difference of coma_H, Sph_Ab, and high_RMS did not vary significantly with eccentricity in our measured data. Similar to the LCA data, the simulated chromatic differences [see Fig. 4(b)] agree well in trend and magnitude of variation with our measured chromatic data, except for Sph_ab for which the sign is reversed.

\section{Simulation of the Chromatic Sources}

To investigate the possible sources that could have an impact on peripheral chromatic aberrations, several factors were tested using the model eye. The first factor was the size of the vitreous chamber. We increased its length to have an eye with $4 \mathrm{D}$ of foveal myopia (myop). The second factor was the GRIN of the crystalline lens. We replaced it with a medium with uniform refractive index. Two uniform refractive indicess were investigated: 1.45 (noGRIN1) and 1.38 (noGRIN2) at reference wavelength $550 \mathrm{~nm}$. For both cases the dispersion equation was calculated using the method suggested by Atchison and Smith [24]. A third factor evaluated was the shape of the retina: an oblate retinal shape, conic constant of -1 (obl), and a prolate retinal shape, conic constant of 0.9 (prol). The fourth factor was the vignetting by the pupil. Eliminating the
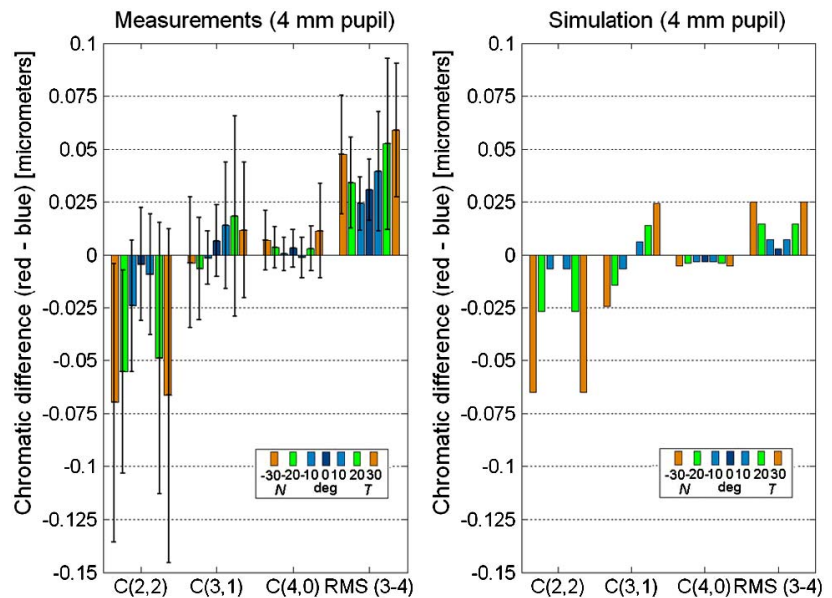

Fig. 4. (Color online) (a) Left figure contains the chromatic difference (red-blue) as function of eccentricity for the measured data. The mean values over all subjects (11) for Zernike coefficients $C_{2}^{2}$, $C_{3}^{1}$, and $C_{4}^{0}$ and high_RMS (third-order and fourth-order) are plotted in micrometers. The error bars show the standard deviation over the 11 subjects. (b) Same data as the left figure but calculated from the simulations. $\mathrm{N}$ stands for nasal and $\mathrm{T}$ for temporal retina. pupil in the eye model allows us to calculate the Zernike coefficients from the entire pupil (noELPS). The fifth and last factor analyzed was the TCA: by adjusting the angles and decentration for each wavelength it is possible to eliminate the impact of the TCA (noTCA). Figure 5(a) shows how M (calculated for the green wavelength $532 \overline{\mathrm{nm}}$ ) varies with eccentricity $\left(0^{\circ}\right.$ to $\left.40^{\circ}\right)$ when changing the different analyzed factors. The changes occur not only in the value of the foveal $\mathrm{M}$ but also the relative peripheral refraction varies with off-axis angle [the lines of the original (org) case, the noTCA case, and the noELPS case are so similar that no distinction can be seen in this graph]. Figure 5(b) shows the LCA (red-blue) for the different cases discussed above. As can be seen, changes in all of the above investigated factors still generate higher LCA with increasing eccentricity. The impact of the noTCA and noELPS cases can hardly be distinguished from the original model (org). The myopic case and the noGRIN cases change the initial value of LCA slightly. But it is only the shape of the retina that has some impact on how LCA changes with eccentricity; a slightly stronger increase was found for the oblate case and slightly less increase for the prolate case. But the effect is marginal compared to the difference in refractive state of the eye and we therefore conclude that the origin of the increase in LCA with eccentricity cannot be explained by any of the five investigated factors.

\section{DISCUSSION}

In this study we have found that the ocular wavefront aberrations vary differently with wavelength depending on the retinal eccentricity. The differences have significance when converting between wavelengths for measurements of the mean spherical equivalent (M) and the horizontal astigmatism (J0) in off-axis angles larger than $20^{\circ}$ from the visual axis. Rynders et al. [28] studied the variation of LCA as a function of eccentricity using the double-pass method. Similar to our findings, they measured on-axis an average LCA of $1 \mathrm{D}$ between 632.8 and $458 \mathrm{~nm}$. With increasing eccentricity they also
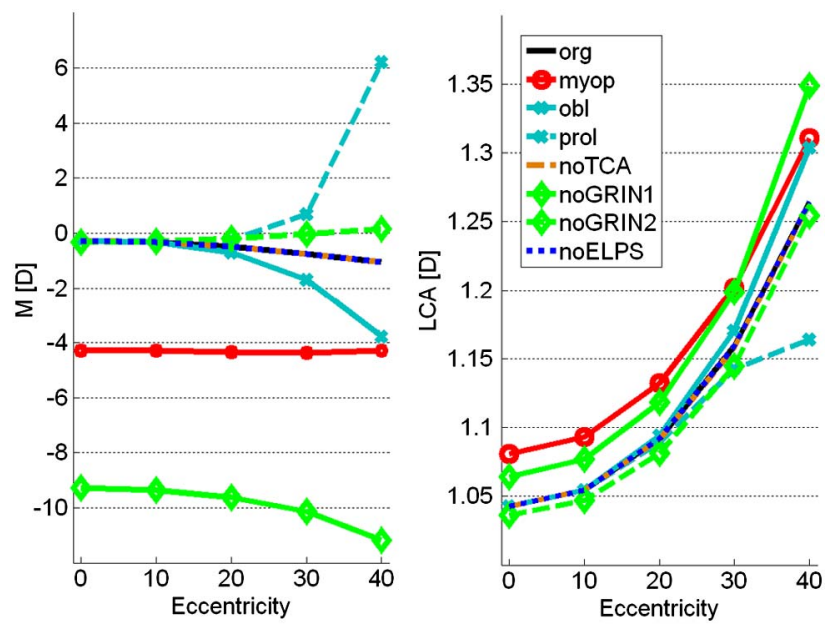

Fig. 5. (Color online) (a) Variation of M with eccentricity at $532 \mathrm{~nm}$ for the different simulated cases: original model eye (org), 4 D myopic eye (myop), retinal conic constant of -1 (obl), retinal conic constant of 0.9 (prol), compensation for TCA (noTCA), uniform distribution of refractive index of the crystalline lens with nref $=1.45$ (noGRIN1) and nref $=1.38$ (noGRIN2), and the use of the whole wavefront information to fit Zernike coefficients (noELPS). (b) LCA (red-blue) for the different simulated cases. All show an increase with eccentricity. 
measured an increase of LCA. The rate of increase was estimated to $0.0126 \pm 0.0092 \mathrm{D} / \mathrm{deg}$ (mean \pm standard deviation). If the increase is linear this would be $0.378 \pm 0.276 \mathrm{D}$ at $30^{\circ}$ off-axis, which is higher than our measured increase in LCA of $0.18 \mathrm{D}$ for $30^{\circ}$ on the nasal retina and $0.31 \mathrm{D}$ for $30^{\circ}$ on the temporal retina.

Because we found a significant difference with eccentricity of some of the chromatic aberrations (M and J0), it encouraged us to look for the possible origin of this variation. Using the model eye, we explored various factors that could have an impact. The simulations show that even drastic changes in path length of the chief ray and in refractive index, which cause large differences of $\mathrm{M}$, have only marginal impact on the LCA (see Fig. 5). The increase of LCA with eccentricity therefore seems to be related to the refractive surfaces and their difference in oblique refractive power for different wavelengths. To examine the impact of the oblique refractive power we simplified the model eye to a one-surface model, with two different shapes of the surface (corresponding to the cornea). When using a spherical surface, Coddington's equations for calculating the oblique power are valid [27]. As a first test, we calculated the LCA using the induced vergence method as described by Campbell [26], extending it with the change of oblique power of the surface for the off-axis angles. As a second test, we did ray tracing in the Indiana reduced model eye ( $p=0.6$ and $z=2.75$ ). Figure 6 (a) compares the off-axis LCA found from the ray tracing through the Liou and Brennan model eye simulation (org), the Indiana reduced model eye simulation (indiana), and the results of the simple approximation (sph sim). All three models show an increase of LCA with eccentricity assuring that the origin of this increase is indeed the difference in oblique refractive power. However, as expected and as can be seen in Fig. 6(a), the predicted values of the LCA for the one-surface models differ from that of the Liou and Brennan model eye, for which the results fall within on standard deviation of the measured values; nevertheless, the variation with angle of the Indiana eye is similar in magnitude. Figure $6(\mathrm{~b})$ shows the variation in chromatic difference with eccentricity for astigmatism, coma, spherical
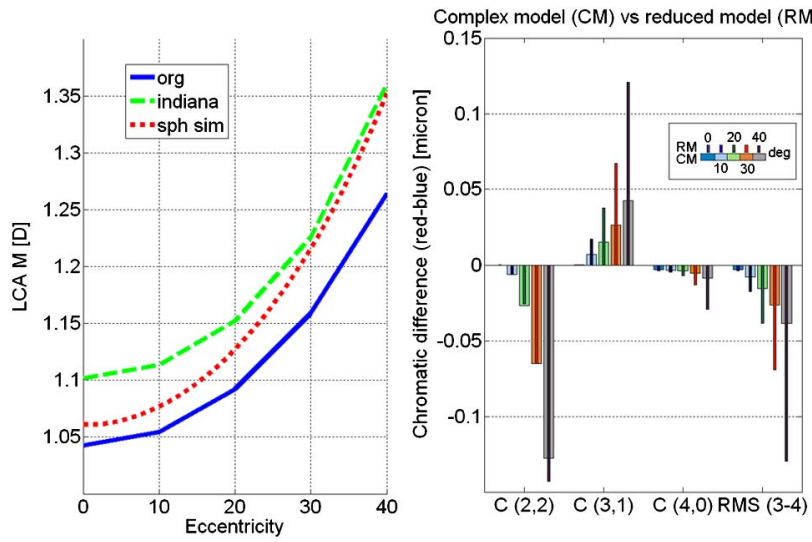

Fig. 6. (Color online) (a) Variation of LCA with eccentricity for three different model eyes. The Liou and Brennan model eye (org), the Indiana reduced model eye (Indiana), and the simple one spherical surface model eye (sph sim). All three predict an increase of LCA with eccentricity, which is the result of the increase of the oblique power of the surface. (b) Variation of the other chromatic aberrations with eccentricity simulated with the complex model eye (CM, narrow colored bars) and the reduced Indiana model eye (RM, wide colored bars). The difference is in micrometers and calculated for a $4 \mathrm{~mm}$ pupil. aberration, and higher-order RMS, calculated both with the complex model eye and the Indiana reduced model eye. As can be seen, the reduced model eye represents a good approximation of the chromatic variation with eccentricity for astigmatism.

The increase of chromatic difference with eccentricity we found suggests that it would be insufficient to compensate chromatic aberrations with a constant offset correction of $M$ when converting off-axis measurements. In clinical applications most wavefront sensors operate in near-infrared. Converting the measured results to a green-yellow wavelength requires a similar order of magnitude of correction as the difference between red and blue. To investigate whether taking into account this variation of chromatic aberration with eccentricity improves the chromatic conversion, we used our modified Liou and Brennan model eye given in Appendix A to estimate the red measurements from the blue measurements (new method) and compared it with an estimation when only compensating defocus with an offset (old method). A small improvement in favor of the new method was found for parameters $\mathrm{M}$ and J0 (mean Bland-Altmann plot $[D]: 0.02 / 0.07,0.01 / 0.05$ (new/old); $95 \%$ limits of agreement $[D]: \pm 0.39 / \pm 0.41, \pm 0.12 / \pm 0.14$; slope regression function: $0.991 / 0.988,1.001 / 1.050)$. The Bland-Altmann plot for $\mathrm{M}$ and J0 is shown in Fig. 7. For Coma_H, Sph_Ab, and high_RMS no difference was found (mean Bland-Altmann plot $[D]: 0.01 / 0.01,0.01 / 0.01,0.00 /-0.01$ (new/old); $95 \%$ limits of agreement $[D]: \pm 0.06 / \pm 0.06, \quad \pm 0.03 / \pm 0.03$, $\pm 0.05 / \pm 0.04$; slope regression function: $0.944 / 1.052,1.216 /$ $1.215,0.971 / 1.051)$.

To examine statistically both methods of conversion for $\mathrm{M}$ and J0, the estimated values were compared to the measured values using paired $t$ tests. A $p$ value larger than 0.05 means that there is no significant difference between the measured data and the estimated data. The results are given in Table 2. The second row of the table compares all estimated data with the measured data for all eccentricities except for $0^{\circ}$ because the methods do not differ there. It can be seen that while for the old method the estimated data is significantly different from the measured data, this is not the case using the new conversion method. Detailed analysis show that the new conversion method gives a better estimation of $\mathrm{M}$ for eccentricities larger than $10^{\circ}$ compared to the old method. For J0,
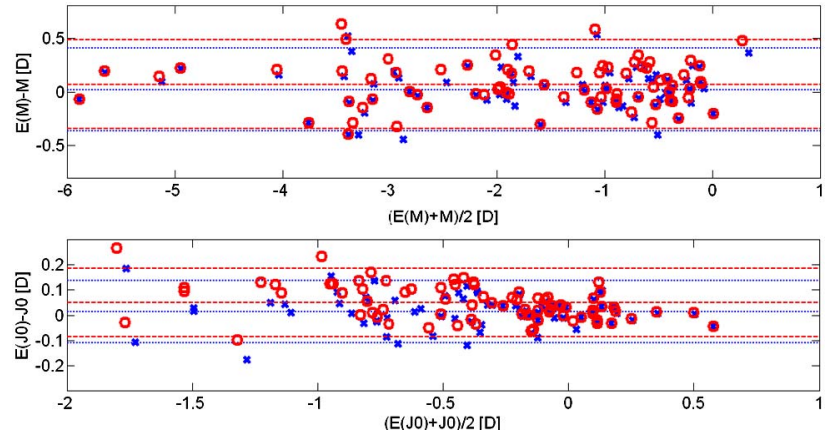

Fig. 7. (Color online) Bland-Altmann plot comparing the estimation of the red data from the blue data using only a foveal offset (circles and striped lines) with the estimation using the new method (crosses and dotted lines). The outermost lines give the $95 \%$ limits of agreement and the central line gives the mean. The letter $\mathrm{E}$ indicates the estimated value. 
Table 2. Results of Paired $t$ Tests Comparing If the Estimated Red Value Differs Significantly from the Measured Red Value Using the New and the Old Conversion Method

\begin{tabular}{ccc}
\hline New/Old & $\mathrm{M}$ & J0 \\
\hline ALL but $0^{\circ a}$ & $0.183 / 0.001$ & $0.061 / 0.000$ \\
$10^{\circ b}$ & $0.550 / 0.790$ & $0.126 / 0.017$ \\
& $(\mathrm{~N}: 0.836 / 0.264 / / \mathrm{T}: 0.126 / 0.182)$ & $(\mathrm{N}: 0.080 / 0.003 / / \mathrm{T}: 0.756 / 0.310)$ \\
$20^{\circ b}$ & $0.098 / 0.006$ & $0.046 / 0.000$ \\
& $(\mathrm{~N}: 0.851 / 0.196 / / \mathrm{T}: 0.046 / 0.009)$ & $(\mathrm{N}: 0.810 / 0.006 / / \mathrm{T}: 0.284 / 0.030)$ \\
& $0.298 / 0.004$ & $0.832 / 0.000$ \\
& $(\mathrm{~N}: 0.399 / 0.005 / / \mathrm{T}: 0.118 / 0.009)$ & $(\mathrm{N}: 0.052 / 0.000 / / \mathrm{T}: 0.949 / 0.019)$ \\
\hline
\end{tabular}

${ }^{a}$ This row gives the comparison of the estimated data with the measured data for all angles expect for $0^{\circ}$. The first value is the $p$ value of the new method compared with the measured data and the second value is the comparison of the old method with the measured data.

${ }^{b}$ These rows give the results of the paired $t$ tests for all data at the given angle and detailed for the nasal $(\mathrm{N})$ and temporal $(\mathrm{T})$ data separately.

nasally all angles and temporally all angles larger than $10^{\circ}$ were better converted using the new method.

Our findings are important mainly in two situations: when comparing different wavelengths and when aberrations are compared at different angles of eccentricity. Significant differences for $\mathrm{M}$ and $\mathrm{J} 0$ were already found for angles of $10^{\circ}$ and $20^{\circ}$. In the case of eyes with relative peripheral myopia, the increase of myopia with angle would be underestimated when measured in near-infrared instead of visible light. The importance of angular chromatic correction in clinical measurements is a function of various factors: the dynamics of the aberration of the subject, the absolute amount of aberrations, the method of measuring, the angular range over which the aberration was measured, and the wavelength of light that was used for the measurements. We found that chromatic aberration change with eccentricity, especially for M and J0, at angles larger than $20^{\circ}$. Although the change of oblique power with eccentricity, which is observed in most human eyes, was found to be the main mechanism behind the angular chromatic difference, slight intersubject variation was observed, which can have some impact on the found trends in individuals.

\section{CONCLUSIONS}

We presented a new wavelength tunable peripheral HS wavefront sensor and a ray-tracing modeling to investigate the offaxis chromatic aberrations in the human eye. We confirmed earlier findings that on-axis measurements at different wavelengths show a wavelength-dependent offset only. In addition, differences were found of chromatic aberration at different angles of eccentricity for both defocus and astigmatism. There was a small, but significant increase of chromatic aberration for the red and blue end of the spectrum and simulations suggest an even larger increase at higher angles of eccentricity. The origin of this change was found to be the oblique refractive power of the surfaces of the eye. According to our simulations, changes in axial length and refractive index profile have little impact on the peripheral chromatic aberrations. Even if only defocus is of interest, the use of a constant offset would introduce an increasing error with eccentricity especially for eccentricities of $20^{\circ}$ and higher. Ray tracing in a model eye, as shown in the current study, can provide a more detailed description of the chromatic aberration profile in the periphery. This type of information could be eventually used for accurate wavelength compensation of off-axis data obtained with state-of-the-art scanner peripheral wavefront sensors [35]. Ray tracing in a model eye, as the one shown in the article, could provide a more suitable chromatic conversion.

\section{APPENDIX A}

In the computer simulations, we flipped the model eye placing the object plane on the retina of the model eye. To maintain the optical properties, various values defining the geometry of the model eye as defined in [33] had to be refit. The dispersion equations of [33] gave insufficient on-axis LCA and were therefore changed with the dispersion equations from [11]. Because the refractive index for the reference wavelength remained the same, the optical properties of the model eye for the reference wavelength did not change. The Cauchy dispersion equations suggested by [11] had to be recalculated to the Schott dispersion equations and a simplified Sellmeier dispersion function to be used in Zemax for the standard surfaces and the gradient five surfaces, respectively. The gradient index is defined with the following equation:

$$
n_{\text {ref }}=n_{0}+n_{r 2} r^{2}+n_{r 4} r^{4}+n_{z 1} z+n_{z 2} z^{2}+n_{z 3} z^{3}+n_{z 4} z^{4},
$$

with

$$
r=\left(x^{2}+y^{2}\right)^{1 / 2} .
$$

Schott dispersion equation:

$$
n(\lambda)^{2}=a_{0}+a_{1} \lambda^{2}+a_{2} \lambda^{-2}+a_{3} \lambda^{-4}+a_{4} \lambda^{-6}+a_{5} \lambda^{-8} .
$$

The simplified Sellmeier dispersion equation:

$$
n(\lambda)^{2}=n\left(\lambda_{\text {ref }}\right)^{2}+\sum_{i=1}^{3} \frac{K_{i}\left(\lambda^{2}-\lambda_{\text {ref }}^{2}\right)}{\lambda^{2}-L_{i}},
$$

with

$$
K_{i}=\sum_{j=1}^{2} K_{i j}\left(n_{\mathrm{ref}}\right)^{j-1}
$$

The coefficients of the different equation can be found in Tables 3-5.

The settings for the FA, visual angle, and decentration used with the original Liou and Brennan model eye for the different angles of eccentricity are given in Table 6 . These settings were obtained for a reference wavelength of $550 \mathrm{~nm}$. 
Table 3. Surface Information and Values of Coefficients Used in the Model Eye for the Liou and Brennan Eye Model Geometry

\begin{tabular}{|c|c|c|c|c|c|c|c|}
\hline Surface & Туре & Name Surface & Radius & Asphericity & Thickness & $\mathrm{N}$ at $555 \mathrm{~nm}$ & \\
\hline 1 & standard & retina & 12.00 & 0 & 16.27 & 1.336 & \\
\hline 2 & gradient 5 & $\begin{array}{l}\text { posterior } \\
\text { crystalline }\end{array}$ & 8.10 & 0.96 & 2.43 & $\operatorname{grad} \mathrm{P}$ & \\
\hline 3 & gradient 5 & medium crystalline & infinity & 0 & 1.59 & $\operatorname{grad} \mathrm{A}$ & \\
\hline 4 (pupil) & standard & anterior crystalline & -12.40 & -0.94 & 3.16 & 1.336 & \\
\hline 5 & standard & posterior cornea & -6.40 & -0.6 & 0.5 & 1.376 & \\
\hline \multirow[t]{2}{*}{6} & standard & anterior cornea & -7.77 & -0.18 & - & - & \\
\hline & $n_{0}$ & $n_{r 2}$ & $n_{r 4}$ & $n_{z 1}$ & $n_{z 2}$ & $n_{z 3}$ & $n_{z 4}$ \\
\hline Grad P & $1.368 E+00$ & $-1.978 E-03$ & 0 & $3.210 E-02$ & $-6.605 E-03$ & 0 & 0 \\
\hline Grad A & $1.407 E+00$ & $-1.978 E-03$ & 0 & 0 & $-1.543 E-02$ & 0 & 0 \\
\hline
\end{tabular}

Table 4. Values of the Schott Dispersion Coefficients Used in the Model Eye

\begin{tabular}{lcccccc}
\hline & $a_{0}$ & $a_{1}$ & $a_{2}$ & $a_{3}$ & $a_{4}$ \\
\hline Cornea & $1.8535 E+00$ & $2.8269 E-04$ & $1.6610 E-2$ & $-1.8719 E-3$ & $1.6283 E-4$ \\
Aqueous humour & $1.7471 E+00$ & $-2.5796 E-4$ & $1.5845 E-2$ & $-1.7850 E-03$ & $1.5126 E-04$ & $7.5807 E-07$ \\
Vitreous humour & $1.7494 E+00$ & $-5.2758 E-4$ & $1.4299 E-2$ & $-1.4114 E-03$ & $1.1750 E-04$ & $8.6476 E-07$ \\
\hline
\end{tabular}

Table 5. Values of the Sellmeier Dispersion Coefficients for a Crystalline Lens

\begin{tabular}{ccccccccc}
\hline$K_{11}$ & $K_{12}$ & $K_{21}$ & $K_{22}$ & $K_{31}$ & $K_{32}$ & $L_{1}$ & $L_{2}$ & $L_{3}$ \\
\hline-543.4493 & 784.8531 & 269.8803 & -389.7629 & 273.6147 & -395.1561 & -0.0010 & 0.0000 & -0.0020 \\
\hline
\end{tabular}

Table 6. Different Values for the Used

Field Angle (FA), Decentration (DEC), and

Visual Angle (VA) for the Original Liou and Brennan Model Eye Simulation ${ }^{a}$

\begin{tabular}{rcr}
\hline $\mathrm{FA}\left[^{\circ}\right]$ & $\mathrm{DEC}[\mathrm{mm}]$ & $\mathrm{VA}\left[^{\circ}\right]$ \\
\hline 26.480 & 2.505 & 40.000 \\
20.090 & 1.753 & 30.000 \\
13.500 & 1.118 & 20.000 \\
6.780 & 0.545 & 10.000 \\
0.000 & 0.000 & 0.000 \\
\hline
\end{tabular}

${ }^{a}$ The values were calculated for a reference wavelength of $550 \mathrm{~nm}$.

\section{ACKNOWLEDGMENTS}

This work was supported by the Ministerio de Ciencia e Innovación, Spain (grants FIS2007-64765, FIS2010-14926, and CSD2007-00033); Fundación Séneca (Region de Murcia, Spain), grant 4524/GERM/06; and by European Commission's sixth framework program through the Marie Curie Research Training Network MYEUROPIA (MRTN-CT-2006-034021).

\section{REFERENCES}

1. F. Schaeffel, A. Glasser, and H. C. Howland, "Accommodation, refractive error and eye growth in chickens," Vision Res. 28, 639-657 (1988).

2. E. L. Smith, C. Kee, R. Ramamirham, Y. Qiao-Grider, and L. Hung, "Peripheral vision can influence eye growth and refractive development in infant monkeys," Invest. Ophthalmol. Visual Sci. 46, 3965-3972 (2005).

3. S. Diether and F. Schaeffel, "Local changes in eye growth induced by imposed local refractive error despite active accommodation," Vision Res. 37, 659-668 (1997).
4. E. L. Smith, L. Hung, and J. Huang, "Relative peripheral hyperopic defocus alters central refractive development in infant monkeys," Vision Res. 49, 2389-2392 (2009).

5. E. L. Smith, L. Hung, J. Huang, T. L. Blasdel, T. L. Humbird, and K. H. Bockhorst, "Effects of optical defocus on refractive development in monkeys: Evidence for local, regionally selective mechanisms," Invest. Ophthalmol. Visual Sci. 51, 3864-3873 (2010).

6. J. Hoogerheide, F. Rempt, and W. P. H. Hoogenboom, "Acquired myopia in young pilots," Ophthalmologica 163, 209-215 (1971)

7. D. O. Mutti, J. R. Hayes, G. L. Mitchell, L. A. Jones, M. L. Moeschberger, S. A. Gotter, R. N. Kleinstein, R. E. Manny, J. D. Twelker, and K. Zadnik, "Refractive error, axial length, and relative peripheral refractive error before and after the onset of myopia," Invest. Ophthalmol. Visual Sci. 48, 2510-2519 (2007).

8. J. Wallman and J. Winawer, "Homeostasis of eye growth and the question of myopia," Neuron 43, 447-468 (2004).

9. L. Lundström, A. Mira-Agudelo, and P. Artal, "Peripheral optical errors and their change with accommodation differ between emmetropic and myopic eyes," J. Vision 9, 17 (2009).

10. L. Lundström, J. Gustafsson, and P. Unsbo, "Population distribution of wavefront aberrations in the peripheral human eye," J. Opt. Soc. Am. A 26, 2192-2198 (2009).

11. D. A. Atchison, N. Pritchard, K. L. Schmid, D. H. Scott, C. E. Jones, and J. M. Pope, "Shape of the retinal surface in emmetropia and myopia," Invest. Ophthalmol. Visual Sci. 46, 1450-1458 (2005).

12. D. O. Mutti, R. I. Sholtz, N. E. Friedman, and K. Zadnik, "Peripheral refraction and ocular shape in children," Invest. Ophthalmol. Visual Sci. 41, 1022-1030 (2000).

13. A. Seidemann, F. Schaeffel, A. Guirao, N. Lopez-Gil, and P. Artal, "Peripheral refractive errors in myopic, emmetropic, and hyperopic young subjects," J. Opt. Soc. Am. A 19, 2363-2373 (2002).

14. P. Artal, A. M. Derrington, and E. Colombo, "Refraction, aliasing and the absence of motion reversals in peripheral vision," Vision Res. 35, 939-947 (1995). 
15. L. Lundström, S. Manzanera, P. M. Prieto, D. B. Ayala, N. Gorceix, J. Gustafsson, P. Unsbo, and P. Artal, "Effect of optical correction and remaining aberrations on peripheral resolution acuity in the human eye," Opt. Express 15, 12654-12661 (2007).

16. J. Santamaría, P. Artal, and J. Bescós, "Determination of the point spread function of human eyes using a hybrid optical-digital method," J. Opt. Soc. Am. A 4, 1109-1114 (1987).

17. A. Guirao and P. Artal, "Off-axis monchromatic aberrations estimated from double pass measurements in the human eye," Vision Res. 39, 207-217 (1999).

18. P. M. Prieto, F. Vargas-Martín, S. Goelz, and P. Artal, "Analysis of the performance of the Hartmann-Shack sensor in the human eye," J. Opt. Soc. Am. A 17, 1388-1398 (2000).

19. D. A. Atchison, D. H. Scott, and W. N. Charman, "Measuring ocular aberrations in the peripheral visual field using HartmannShack aberrometry," J. Opt. Soc. Am. A 24, 2963-2973 (2007).

20. E. J. Fernández and P. Artal, "Ocular aberrations up to the infrared range: from 632.8 to $1070 \mathrm{~nm}$," Opt. Express 16, 21199-21208 (2008).

21. S. Manzanera, C. Canovas, P. M. Prieto, and P. Artal, "A wavelength tunable wavefront sensor for the human eye," Opt. Express 16, 7748-7755 (2008).

22. W. N. Charman and J. A. M. Jennings, "Objective measurements of the longitudinal chromatic aberration of the human eye," Vision Res. 16, 999-1005 (1976).

23. L. N. Thibos, M. Ye, X. Zhang, and A. Bradley, "The chromatic eye: a new reduced-eye model of ocular chromatic aberrations in humans," Appl. Opt. 31-19, 3594-3600 (1992).

24. D. A. Atchison and G. Smith, "Chromatic dispersions of the ocular media of human eyes,” J. Opt. Soc. Am. A 22, 29-37 (2005).
25. J. Nam, J. Rubinstein, and L. N. Thibos, "Wavelength adjustment using an eye model from aberrometry data,” J. Opt. Soc. Am. A 27, 1561-1574 (2010).

26. C. E. Campbell, "Relative importance of sources of chromatic refractive error in the human eye," J. Opt. Soc. Am. A 27 730-738 (2010)

27. Y. Wang and L. N. Thibos, "Oblique (off-axis) astigmatism of the reduced schematic eye with elliptical refracting surface," Optometry Vision Sci. 74, 557-562 (1997).

28. M. C. Rynders, R. Navarro, and M. A. Losada, "Objective measurement of the off-axis longitudinal chromatic aberration in the human eye," Vision Res. 38, 513-522 (1998).

29. I. Escudero-Sanz and R. Navarro, "Off-axis aberrations of a wide-angle schematic eye model," J. Opt. Soc. Am. A 16, 1881-1891 (1999).

30. Y. U. Ogboso and H. E. Bedell, "Magnitude of lateral chromatic aberration across the retina of the human eye," J. Opt. Soc. Am. A 4, 1666-1672 (1987).

31. L. Lundström and P. Unsbo, "Unwrapping Hartmann-Shack images from highly aberrated eyes using an iterative B-spline based extrapolation method," Optometry Vision Sci. 81, 569-577 (2004).

32. American National Standards Institute, "Methods for reporting optical aberrations of eyes," Tech. Rep. ANSI Z80.28-2004 (2004).

33. H. Liou and N. A. Brennan, "Anatomically accurate, finite model eye for optical modeling,” J. Opt. Soc. Am. A 14, 1684-1695 (1997)

34. D. A. Atchison, N. Pritchard, and K. L. Schmid, "Peripheral refraction along the horizontal and vertical visual fields in myopia," Vision Res. 46, 1450-1458 (2006).

35. B. Jaeken, L. Lundström, and P. Artal, "Fast scanning peripheral wave-front sensor for the human eye," Opt. Express 19 7903-7913 (2011). 